http: //lcawww.epfl.ch/Publications/LeBoudec/LeBoudecV04.pdf

\title{
Understanding the Simulation of Mobility Models with Palm Calculus
}

\author{
Jean-Yves Le Boudec
}

June 23, 2005

\begin{abstract}
The simulation of mobility models such as the random waypoint often cause subtle problems, for example the decay of average speed as the simulation progresses, a difference between the long term distribution of nodes and the initial one, and sometimes the instability of the model. All of this has to do with time averages versus event averages. This is a well understood, but little known topic, called Palm calculus. In this paper we first give a very short primer on Palm calculus. Then we apply it to the random waypoint model and variants (with pause time, random walk). We show how to simply obtain the stationary distribution of nodes and speeds, on a connected (possibly non convex) area. We derive a closed form for the density of node location on a square or a disk. We also show how to perform a perfect (i.e. transient free) simulation without computing complicated integrals. Last, we analyze decay and explain it as either convergence to steady state or lack of convergence.
\end{abstract}

Keywords: Mobility Model, Random Waypoint, Random Walk, Palm Calculus, Perfect Simulation.

\section{Introduction}

This paper reviews recent results about the random waypoint model; we give a short tutorial on Palm calculus, then we show how it helps understanding the simulation of the random waypoint. We also derive some new results like a closed form for the probability density function of the node location.

The simulation of the random waypoint model poses a surprising number of challenges, such as:

- "Unfortunately, the vast majority of mobility models [...] suffer from decay; average speed decreases until converging to some long-term average. Such decay provides an unsound 
basis for simulation studies that collect results averaged over time, complicating the experimental process" [15].

- "The random waypoint model is considered harmful" [14]. The "harmfulness" of random waypoint can be attributed to the uniform selection of speed over the interval $\left[v_{\min }, v_{\max }\right]$, with $v_{\min }=0$. Taking $v_{\min }>0$ solves the problem.

- Simulation of some random waypoint models can be started in steady-state [8, 12]

- The distribution of nodes is not uniform [4]

These questions have been addressed in an ad-hoc fashion in the literature. The derivations involve long and complex computations, leaving the reader with little understanding of the "why". Our starting point is the observation that all of this can be very easily understood with a little bit of Palm calculus. Palm calculus is a set of formulas that relate time averages to event averages. They are now well established, but not widely used or even known in applied areas.

The reason is maybe that the construction of the Palm theory is complex in continuous time, whereas in discrete time it is a simple exercise on conditional probabilities $[1,7]$. In this paper we explain Palm calculus concisely, and rigorously in discrete time.

Then we show how to apply Palm calculus to do simulation "the right way" for mobility models. In particular, we show how to easily compute the stationary distribution of nodes in a random waypoint model on any convex area (a problem considered difficult, or even intractable in [4]). We also show how to easily write a simulation that is in stationary regime at time 0 (this is called a "perfect" simulation). Last, we can also understand when the random waypoint model has at all a stationary regime and when not.

Being able to simulate the stationary distribution of a mobility model is important for two reasons. First, this speeds up considerably the warmup phase of a simulation (if we use a perfect simulation of the mobility model). As illustrated on Figure 10, the transient time for a realistic mobility model may span several hundreds of seconds of simulated time, which is large compared to the transients of simulations of networking protocols without mobility models. Second, this is useful when comparing the performance of some system with or without mobility; many published papers invariably distribute nodes uniformly in the static scenario, whereas, as we see later in this paper, the stationary distribution of nodes for a mobile scenario with the random waypoint is not uniform. A fair comparison should instead place the nodes for the static scenario according to the stationary distribution of the mobile scenario.

There are well established techniques for performing perfect simulation. The method in [9] applies to a large class of Markov chains on which some partial ordering can be defined, and uses coupling from the past (sample trajectories starting in the past at different initial conditions). The technique presented in this paper is much simpler, as, unlike in the case of [9], we can obtain an explicit representation of the stationary distribution.

Our use of Palm calculus simplifies the understanding of existing results on mobility models. It also extends them in a few directions. First we show that the stationary distribution can be computed explicitly for a wide class of models, including any arbitrary convex area. Second, we find how to do perfect simulation, which involves a little more than simply finding the stationary distribution of locations and speeds. We show how to sample the stationary regime without computing difficult integrals. In order to keep the focus on the main ideas, rather than clumsy technical details, we first start with the simplest random waypoint model, without pauses, on a convex area. The 
generalization to variants (with pause times or random walk with wrapping) is given at the end of the paper.

The rest of this paper is organized as follows. In Section 2 we describe the simplest random waypoint model. In Section 3 give a brief tutorial on Palm calculus. In Section 4 we apply Palm calculus to obtain the stationary distribution of nodes and speeds, and to perfect simulation. In Section 5 we analyze when the random waypoint model has a stationary regime and when not. In Section 6 we generalize the results to variants of the random waypoint model: random waypoint with pauses, on non convex (but connected) area, random walk with reflection or wrapping.

\section{Notation And Terms}

$\begin{array}{ll}A & \text { Geographical area over which random waypoint is defined } \\ a_{M}(\theta) & \text { Distance from } M \in A \text { to the boundary of } A \text {, in the direction } \theta \text { (Figure } 8 \text { ) } \\ D_{n} & \text { Distance }\left\|M_{n+1}-M_{n}\right\| \\ \bar{\Delta} & \text { Average distance between two points in } A \\ \mathbb{E}^{0}\left(X_{0}\right) & \text { Palm expectation, event stationary expectation: } \\ & \text { expectation of the stationary process } X_{t} \text { sampled at an arbitrary transition time } T_{n} \\ \mathbb{E}_{p a}^{0}\left(X_{0}\right)\left[\mathbb{E}_{m o}^{0}\left(X_{0}\right)\right] & \text { Expectation at an arbitrary beginning of pause [move] interval } \\ f_{p a}^{0} & \text { Density of distribution of pause time } \\ f_{T}^{0}(t) & \text { Density of distribution of trip duration (random walk) } \\ f_{V}^{0}(v) & \text { Density of distribution of speed } V_{n} \text { selected at waypoint } \\ f_{\vec{V}}^{0}(\vec{v}) & \text { Density of distribution of speed vector (random walk) } \\ K_{2} & =1 /\left(\text { area }(A)^{2} \bar{\Delta}\right) \\ \lambda & \text { Intensity: number of transitions per time unit } \\ \lambda_{p a}\left[\lambda_{m o}\right] & \text { Number of transitions per time unit in a model with only pauses [moves] } \\ M(t)\left[M_{n}\right] & \text { Position of mobile at time } t \text { [just after } n \text {th transition] } \\ \text { Next }(t) & \text { Position at the next transition after time } t \\ \operatorname{Perfect} \text { Simulation } & \text { Mobility model is in stationary regime at all times } \\ \operatorname{Prev}(t) & \text { Position at the next transition before or at time } t \\ T_{n} & \text { Selected transition time (time at which a waypoint is reached or pause is finished) } \\ R(t) & \text { Remaining time until next transition } \\ \text { Uniform Speed } & f_{V}^{0}(v)=\frac{1}{v_{\max }-v_{\min }} 1_{\left\{v_{\min }<v<v_{\max }\right\}} \\ V(t)\left[V_{n}\right] & \left.\text { Numeric speed at time } t \text { [in interval }\left[T_{n}, T_{n+1}\right)\right] \\ \vec{V}(t)\left[\vec{V}_{n}\right] & \left.\text { Speed vector at time } t \text { [in interval }\left[T_{n}, T_{n+1}\right)\right] \\ \Phi(t)\left[\Phi_{n}\right] & \left.\text { Phase (pa or } m o \text { ) of mobile at time } t \text { [in interval }\left[T_{n}, T_{n+1}\right)\right] \\ w & \text { Wrapping function (random walk model) } \\ & \end{array}$

\section{Random Waypoint without Pauses on Arbitrary Area}

\subsection{Definition of Random Waypoint}

We start by defining the random waypoint without pauses on an arbitrary convex and bounded area $A$. Generalization to models with pause times, reflections, wrapping or connected but non convex areas is given in Section 6 . 
The model is defined as follows (Figure 1). It defines the movement of one mobile in some area $A$. A common example for $A$ is a rectangle or a disk. The mobile moves from one "waypoint" $M_{n}$ to the next $M_{n+1}$ according to the following rules.

- The next waypoint $M_{n+1}$ is chosen uniformly in the area $A$, independent of past and present events;

- The next speed $V_{n}$ is chosen according to a probability density function $f_{V}^{0}(v)$, independent of past and present events; a common example ("uniform speed") is to choose $V_{n}$ uniformly between $v_{\min }$ and $v_{\max }$, i.e. $f_{V}^{0}(v)=\frac{1}{v_{\max }-v_{\min }} 1_{\left\{v_{\min }<v<v_{\max }\right\}}$;

- The mobile moves towards $M_{n+1}$ at the constant speed $V_{n}$.

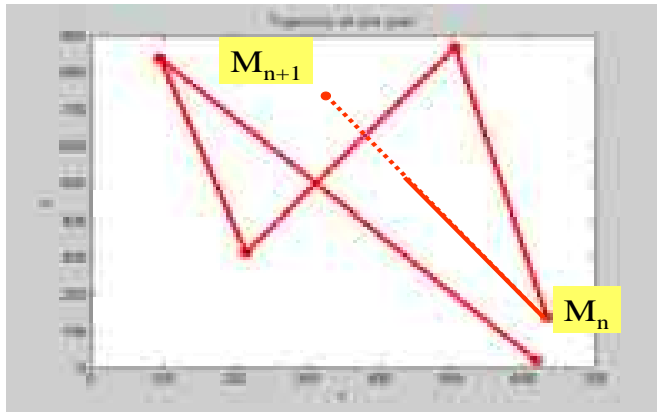

(a)

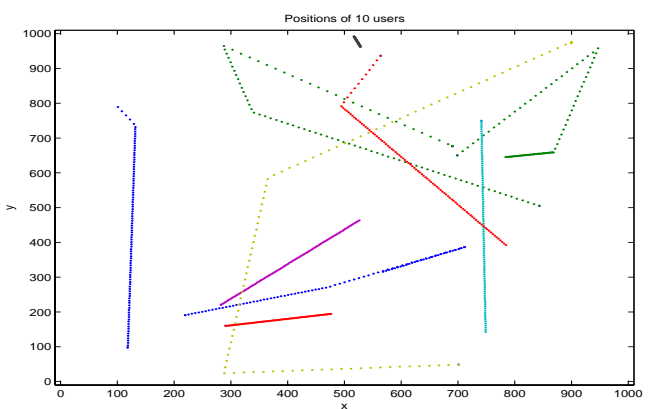

(b)

Figure 1: (a) Definition of Random Waypoint when the area $A$ is a rectangle. (b) Simulation with 10 independent mobiles

To fully define the model, we need to specify the initial condition. We consider first the common practice that is to start the mobile at a waypoint, i.e. pick $M_{0}$ uniformly in $A$ and choose the next waypoint and speed $V_{0}$ as above. We will consider better choices in Section 4.

\subsection{Features of Random Waypoint}

If we simulate the model we have just defined, a number of striking observations can be made.

Different Speed Distributions The speed is initially chosen from the distribution with density $f_{V}^{0}$, however, as the simulation progresses, this distribution is altered [15]. Figure 2 displays a histogram of instantaneous speeds, sampled at arbitrary instants of a long simulation with many independent mobiles, versus the histogram of speeds at waypoints (which has the same distribution as the initial speed). We see that it is more likely to find a mobile with a small speed than indicated from for the distribution with density $f_{V}^{0}$. An intuitive explanation is that a mobile spends more time at lower speed, therefore it is more likely to be sampled at low speed. We will see in Section 4 that the exact form of the histogram in Figure 2 can easily be predicted with Palm calculus.

Different Distributions of Mobile Positions The initial mobile position is uniform in the area $A$, however, with time, the distribution of mobile positions tends to be more dense towards the 


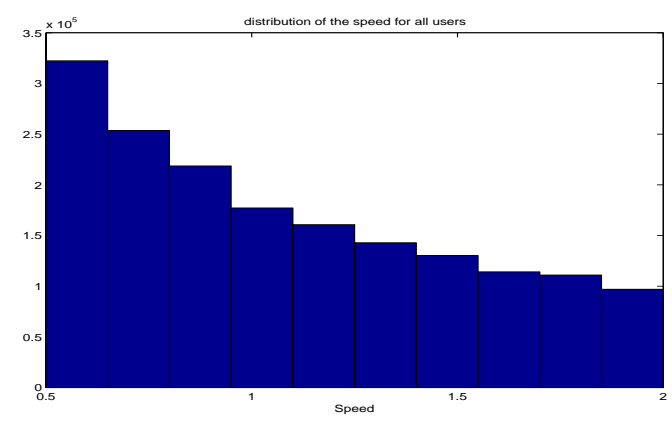

(a) Arbitrary Time Instant

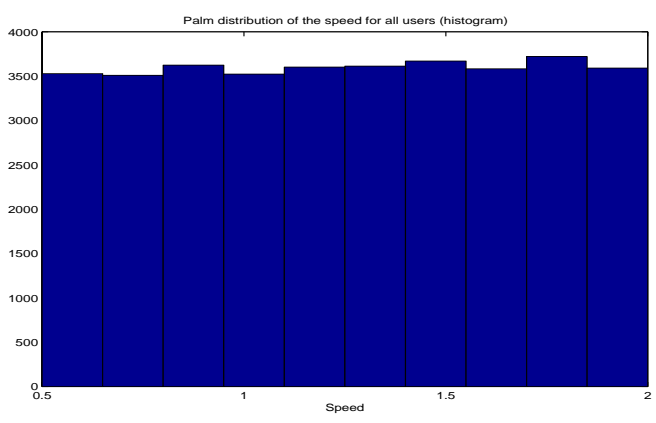

(b) Arbitrary Waypoint

Figure 2: Histogram of speeds sampled uniformly over one day (a) or at waypoints (b). There are 200 independent mobiles, the density $f_{V}$ is uniform between $v_{\min }$ and $v_{\max }$ with $v_{\min }=0.5 \mathrm{~m} / \mathrm{s}, v_{\min }=2 \mathrm{~m} / \mathrm{s}$. $A$ is a square area with size $1000 \mathrm{~m}$.

middle of the area (Figure 3). We will see in Section 4 that is is simply the convergence towards steady state, and the distribution of nodes can simply be computed.

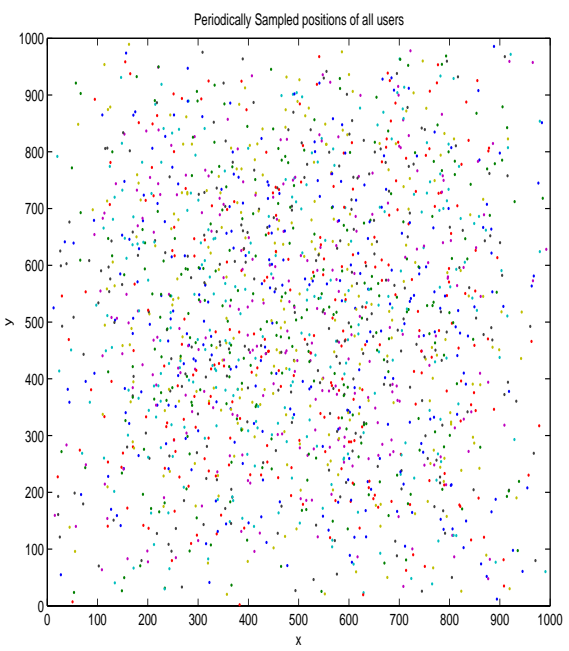

(a) Arbitrary Time Instant

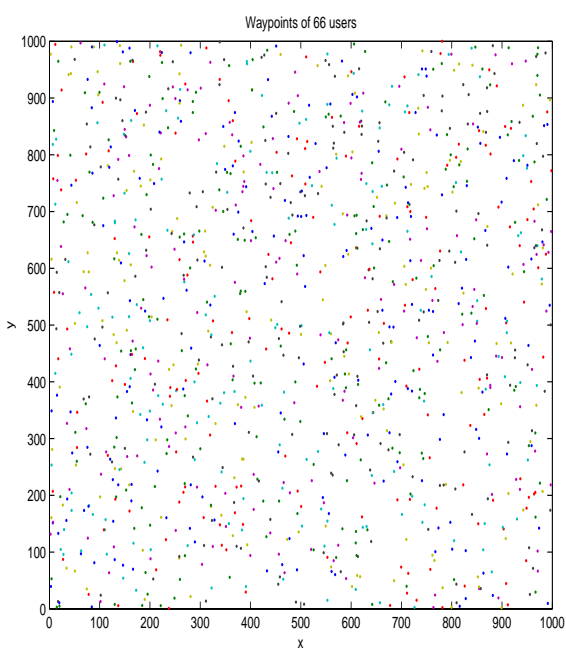

(b) Arbitrary Waypoint

Figure 3: Nodes positions sampled uniformly (a) or at waypoints (b). Same parameters as in Figure 2.

Speed Decay The average speed at time $t$ is defined as the distance run by a mobile, divided by $t$. As the simulation progresses the average speed decays. When the density $f_{V}$ is uniform between $v_{\min }$ and $v_{\max }$, there seems to be convergence to a positive speed limit if $v_{\min }>0$; if $v_{\min }=0$, empirical results are less clear Figure 4. We will see in Section 5 that for $v_{\min }>0$ the average speed decays to a limit equal to the stationary expectation of the speed (the mean of the histogram in Figure 2 (a)); in contrast, for $v_{\min }=0$, the model is theoretically unstable, i.e. the average speed does decay to 0 , but this cannot be observed in practice. 


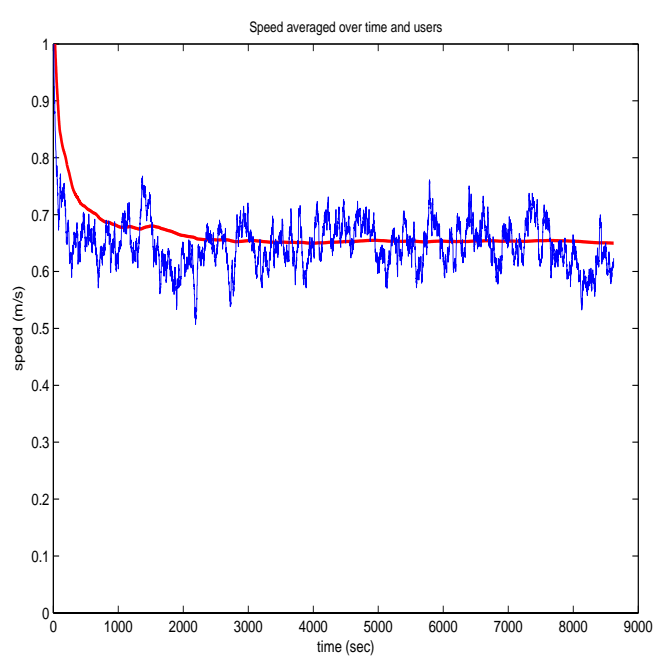

(a) $v_{\min }=0.1 \mathrm{~m} / \mathrm{s}$

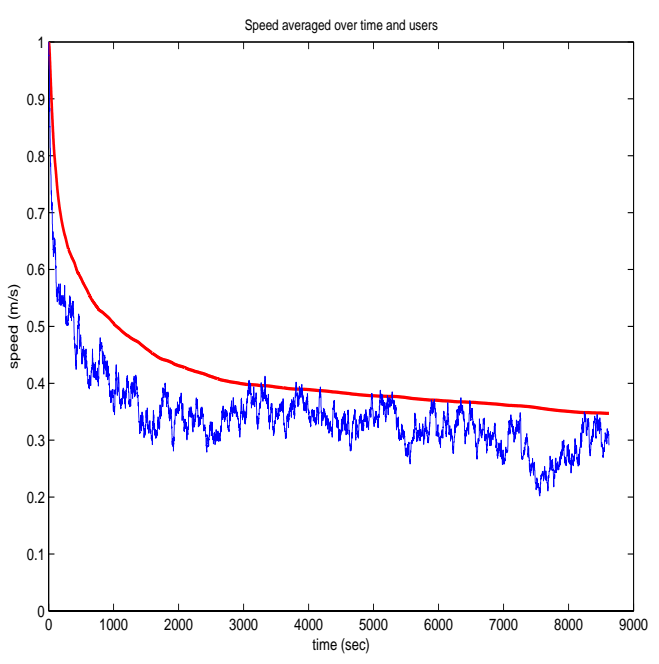

(b) $v_{\min }=0 \mathrm{~m} / \mathrm{s}$

Figure 4: Speed averaged over time and users (thick line) and instant speed averaged over users (thin line). Other parameters same as Figure 2.

\section{A Palm Calculus Primer}

Palm calculus is a set of formulas that relate time averages versus event averages. "Time averages" are obtained by sampling the system at arbitrary time instants; this corresponds to "time stationary" distributions ${ }^{1}$. For example, the distribution of points on Figure 3 (a) is obtained by sampling the system every $10 \mathrm{sec}$ of simulated time. The "event average" viewpoint is obtained by sampling the system when selected state transitions occur. For example, Figure 3 (b) is obtained by sampling the system when a mobile reaches a waypoint. The distribution obtained in this way is called a Palm distribution; this is the same as "event stationary" distribution. In signal processing terminology, this is called adaptive sampling.

Stationarity Palm calculus applies to stationary processes. We assume that we are observing the output of a simulation, which we interpret as a sample of a stochastic process $S_{t}$. This process is stationary if for any any $n$, any sequence of times $t_{1}<t_{2}<\ldots<t_{n}$ and any time shift $u$ the joint distribution of $\left(S_{t_{1}+u}, S_{t_{2}+u}, \ldots, S_{t_{n}+u}\right)$ is independent of $u$. In other words, the process does not change statistically as it gets older.

In practice, stationarity often occurs if we let the simulation run long enough. For example, this is always true of a Markov chain over a finite set space; over an infinite state space, this may require additional stability conditions. For the random waypoint model, there is such a condition; it bears on the distribution of speed (Section 5). If this condition holds, the speed decay as in Figure 4 (a) is simply the symptom of convergence to the stationary regime.

The rest of this section applies to an observation $X_{t}$ made from a simulation output, that is assumed to be "jointly stationary" with the simulation state $S_{t}$ (i.e. $\left(X_{t}, S_{t}\right)$ is a stationary process).

\footnotetext{
${ }^{1}$ also called "stationary distribution" in the literature
} 
Note that even if the simulation is stationary, one might easily define outputs that are not jointly stationary. Examples of jointly stationary observations for the random waypoint are: instantaneous speed, or the time elapsed since last waypoint; a counter-example is the time at which last waypoint occurred.

\section{Time Averages.}

If $X_{t}$ is jointly stationary with the simulation, the distribution of $X_{t}$ is, by definition, independent of $t$; it is called the time stationary distribution of $X$.

If $X_{t}$ is ergodic (which, is for example true on a discrete state space if any state can be reached from any state, given that we assumed that $X_{t}$ is stationary), for any bounded function $\phi$, we can estimate $\mathbb{E}\left(\phi\left(X_{t}\right)\right)$ by (assuming time $t$ is discrete):

$$
\mathbb{E}\left(\phi\left(X_{t}\right)\right) \approx \frac{1}{T} \sum_{t=1}^{T} \phi\left(X_{t}\right)
$$

when $T$ is large. An equivalent statement is, for any set $W$ :

$$
\mathbb{P}\left(X_{t} \in W\right) \approx \text { fraction of time that } X_{t} \text { is in the set } W
$$

In other words, the time stationary distribution of $X_{t}$ can be estimated by a time average. This is how the histograms in Figure 2 (a) were obtained.

Selected Transitions, Point Process and Intensity Consider now a set of "selected transitions" of the simulation, assumed to have reached its stationary regime. By this we mean a random sequence of time instants $T_{n}$, defined as the times at which the simulation $S_{t}$ reaches a certain subset of the state space, or does a transition from some state $s$ to some state $s^{\prime}$, where $\left(s, s^{\prime}\right)$ is in a specified set. For example, $\left(T_{n}\right)_{n \in \mathbb{Z}}$ may be the sequence of times at which a waypoint is reached (we reach a waypoint when $M(t)=N \operatorname{ext}(t)$ ). $T_{n}$ is called a "point process".

Since the simulation is in stationary regime, we imagine that, at time 0 , the simulation has been running for some time. Because the point process of selected transitions is defined in terms of transitions of the simulation state $S_{t}$, it is also stationary. It is convenient, and customary, to take as convention

$$
T_{0} \leq 0<T_{1}
$$

In other words, $T_{0}$ is the last time a transition occurred before time 0 , and $T_{1}$ the next transition time starting from time 0 .

The "intensity" $\lambda$ of the point process of selected transitions is defined as the expected number of transitions per time unit. We assume that there cannot be two transitions at the same point in time. In discrete time, $\lambda$ is simply equal to the probability that there is a selected transition at an arbitrary point in time:

$$
\lambda=\mathbb{P}\left(T_{0}=0\right)
$$

In continuous time, the intensity $\lambda$ is defined as the unique number such that the number $N(t, t+\tau)$ of selected transitions during any interval $[t, t+\tau]$ satisfies [1]:

$$
\mathbb{E}(N(t, t+\tau))=\lambda \tau
$$


Palm Expectation and Palm Probability Let $Y$ be some random output of the simulation, assumed to be integrable (for example because it is bounded). We define the expectation $\mathbb{E}^{t}(Y)$ as the conditional expectation:

$$
\mathbb{E}^{t}(Y)=\mathbb{E}(Y \mid \text { a selected transition occurs at time } t)
$$

If $Y=X_{t}$ where $X_{t}$ and the simulation are jointly stationary, $\mathbb{E}^{t}\left(X_{t}\right)$ does not depend on $t$. For $t=0$, it is called the:

\section{Definition 3.1 (Palm expectation)}

$$
\mathbb{E}^{0}\left(X_{0}\right)=\mathbb{E}\left(X_{0} \mid \text { a selected transition occurs at time } 0\right)
$$

The Palm probability is defined similarly, namely

$$
\mathbb{P}^{0}\left(X_{0} \in W\right)=\mathbb{P}\left(X_{0} \in W \mid \text { a selected transition occurs at time } 0\right)
$$

Note that $\mathbb{P}^{0}\left(T_{0}=0\right)=1$, i.e., under the Palm probability, $T_{0}$ is 0 .

The interpretation of the definition is easy in discrete time, if we assume that the set of selected transitions is "simple", i.e. there cannot be more than one transition at any instant $t$; in this case Equation (2) has to be taken in the usual sense of conditional probabilities:

$$
\mathbb{E}^{t}(Y)=\mathbb{E}(Y \mid N(t)=1)=\frac{\mathbb{E}(Y N(t))}{\mathbb{E}(N(t))}=\frac{\mathbb{E}(Y N(t))}{\mathbb{P}(N(t)=1)}
$$

where $N(t)=1$ if a selected transitions occurs at time $t, 0$ otherwise.

In continuous time, the event "a selected transition occurs at time $t$ " has a 0 probability and cannot be conditioned upon. However, it is possible to give a meaning to such a conditional expectation, similar to the way one can define the conditional probability density function of a continuous random variable:

$$
\mathbb{E}^{t}(Y)=\lim _{\tau \rightarrow 0} \frac{\mathbb{E}(Y N(t, t+\tau))}{\mathbb{E}(N(t, t+\tau))}=\lim _{\tau \rightarrow 0} \frac{\mathbb{E}(Y N(t, t+\tau))}{\lambda \tau}
$$

where the limit is in the Radon-Nykodim sense, defined as follows. For a given random variable $Y$, consider the measure $\mu$ defined for any measurable subset $B$ of $\mathbb{R}$ by

$$
\mu(B)=\frac{1}{\lambda} \mathbb{E}\left(Y \sum_{n \in \mathbb{Z}} 1_{\left\{T_{n} \in B\right\}}\right)
$$

where $\lambda$ is the intensity of the point process $T_{n}$. If $B$ is negligible (i.e. its Lebesgue measure, or length, is 0 ) then, with probability 1 there is no transition in $B$ and $\mu(B)=0$. By the Radon-Nykodim theorem [10], there exists some function $g$ defined on $\mathbb{R}$ such that for any $B: \mu(B)=\int_{B} g(t) d t$. The Palm expectation $\mathbb{E}^{t}(Y)$ is defined as $g(t)$. In other words, for a given random variable $Y, \mathbb{E}^{t}(Y)$ it is defined as the function of $t$ that satisfies, for any $B$ :

$$
\mathbb{E}\left(Y \sum_{n \in \mathbb{Z}} 1_{\left\{T_{n} \in B\right\}}\right)=\lambda \int_{B} \mathbb{E}^{t}(Y) d t
$$

See [1] for a complete treatment of Palm calculus. 
Event Averages If the simulation is such that the ergodic theorem holds then we have

$$
\mathbb{E}^{0}\left(X_{0}\right) \approx \frac{1}{N} \sum_{n=1}^{N} X_{T_{n}}
$$

for $N$ large. An equivalent statement is that

$$
\mathbb{P}^{t}\left(X_{t} \in W\right)=\mathbb{P}^{0}\left(X_{0} \in W\right) \approx \text { fraction of selected transitions at which } X_{t} \text { is in } W
$$

This is how the histograms in Figure 2 (b) were obtained, with $X_{t}=$ speed at time $t$ and a selected transition is a departure from a waypoint. This is why we call a Palm distribution an event stationary distribution.

The Inversion and Intensity Formulas There are many formulas that relate time and event averages. We give here the two most useful ones in the context of random waypoint models. Also known under the name of Ryll-Nardzewski and Slivnyak's formula, the inversion formula relates the time stationary and Palm probabilities.

\section{Theorem 3.1 (Inversion Formula) • In discrete time:}

$$
\mathbb{E}\left(X_{t}\right)=\mathbb{E}\left(X_{0}\right)=\lambda \mathbb{E}^{0}\left(\sum_{s=1}^{T_{1}} X_{s}\right)=\lambda \mathbb{E}^{0}\left(\sum_{s=0}^{T_{1}-1} X_{s}\right)
$$

- In continuous time:

$$
\mathbb{E}\left(X_{t}\right)=\mathbb{E}\left(X_{0}\right)=\lambda \mathbb{E}^{0}\left(\int_{0}^{T_{1}} X_{s} d s\right)
$$

Proof. We give the proof in discrete time only. We show only that $\mathbb{E}\left(X_{0}\right)=\lambda \mathbb{E}^{0}\left(\sum_{s=1}^{T_{1}} X_{s}\right)$, as the second equality is similar. By definition of a conditional probability and of $\lambda$ :

$$
\lambda \mathbb{E}^{0}\left(\sum_{s=1}^{T_{1}} X_{s}\right)=\mathbb{E}\left(\sum_{s=1}^{T_{1}} X_{s} N(0)\right)
$$

Now for $s>0$, the event " $s \leq T_{1}$ " is equivalent to " $N(1, s-1)=0$ " thus

$$
\begin{aligned}
\lambda \mathbb{E}^{0}\left(\sum_{s=1}^{T_{1}} X_{s}\right) & =\mathbb{E}\left(\sum_{s=1}^{\infty} X_{s} N(0) 1_{\{N(1, s-1)=0\}}\right) \\
& =\mathbb{E}\left(\sum_{s=1}^{\infty} X_{0} N(-s) 1_{\{N(1-s, 1)=0\}}\right)=\mathbb{E}\left(X_{0} \sum_{s=1}^{\infty} N(-s) 1_{\{N(1-s, 1)=0\}}\right)
\end{aligned}
$$

where the last line is by stationarity. Let $T^{-}(-1)$ be the most recent time at which a selected transition occured before or at time -1 . This time is finite with probability 1 , by stationarity. We have $N(-s) 1_{\{N(1-s, 1)=0\}}=1$ if and only if $T^{-}(-1)=-s$, thus, with probability 1 :

$$
1=\sum_{s=1}^{\infty} N(-s) 1_{\{N(1-s, 1)=0\}}
$$

which shows the formula. 
Theorem 3.2 (Intensity Formula) The average number of selected transitions per time unit $\lambda$ satisfies

$$
\frac{1}{\lambda}=\mathbb{E}^{0}\left(T_{1}-T_{0}\right)=\mathbb{E}^{0}\left(T_{1}\right)
$$

Proof.Apply the intensity formula with $X_{t}=1$.

Note that the right-handside $\mathbb{E}^{0}\left(T_{1}-T_{0}\right)$ is simply the mean interval between selected transitions. The proof of both intensity and inversion formulae are simple in discrete time, where they are simple exercises on conditional probabilities. In contrast, they are more complicated in continuous time [1] and are not given here.

Example: The Bus Inspector and Feller's Paradox Before returning to the random waypoint, we illustrate the use of the intensity and inversion formulas on a simple example. Assume at a bus stop there pass in average $\lambda$ buses per hour. An inspector measures the time between all bus arrivals. She estimates $\mathbb{E}^{0}\left(T_{1}-T_{0}\right)=\frac{1}{\lambda}$.

Joe arrives at time $t$ and measures $X_{t}=$ ( time until next bus - time since last bus). Joe estimates $\mathbb{E}\left(X_{0}\right)=\mathbb{E}\left(T_{1}-T_{0}\right)$. By the inversion formula, Joe's estimate is

$$
\mathbb{E}\left(T_{1}-T_{0}\right)=\lambda \mathbb{E}^{0}\left(\int_{0}^{T_{1}} X_{t} d t\right)=\lambda \mathbb{E}^{0}\left(T_{1}^{2}\right)=\frac{1}{\lambda}+\lambda \operatorname{var}^{0}\left(T_{1}-T_{0}\right)
$$

where $\operatorname{var}^{0}\left(T_{1}-T_{0}\right)$ is the variance, under the Palm probability, of the time between buses.

Thus, Joe's estimate is always larger than the inspector's by a term equal to $\lambda \operatorname{var}^{0}\left(T_{1}-T_{0}\right)$, although both sample the same system (but not with the same viewpoint). This systematic bias is known as "Feller's paradox". Intuitively, it occurs because a stationary observer (Joe) is more likely to fall in a large interval.

We did not make any assumption other than stationarity about the process of bus arrivals in this example. Thus these results and Feller's paradox are true for any selected transitions of any stationary system. In particular, note that they are true whether the intervals $T_{n+1}-T_{n}$ are independent or not.

Other Palm Calculus Formulas There are many other Palm calculus formulas [1, 7]. Another popular one is "Little's formula" $\lambda R=N$ where $R$ is the Palm expectation of the residence time for customers through an arbitrary stationary system, $\lambda$ is the intensity of the arrivals, and $N$ is the stationary expectation of the number of customers in the system. 


\section{Time Stationary Behaviour and Perfect Simulation}

\subsection{Intensity}

We apply Palm calculus to the random waypoint model. Assume for now that the model has a stationary regime and consider as selected transitions instants $T_{n}$ the times at which waypoints are reached.

Theorem 4.1 The average number of waypoints per time unit $\lambda$ is given by

$$
\lambda^{-1}=\mathbb{E}^{0}\left(D_{1}\right) \mathbb{E}^{0}\left(\frac{1}{V_{0}}\right)=\bar{\Delta} \int_{0}^{\infty} \frac{1}{v} f_{V}^{0}(v) d v
$$

where $\bar{\Delta}$ is the average distance between two points in $A$.

Proof. By the intensity formula (Equation (8))

$$
\lambda^{-1}=\mathbb{E}^{0}\left(T_{1}\right)=\mathbb{E}^{0}\left(\frac{D_{1}}{V_{0}}\right)
$$

with $D_{1}:=\left\|M_{1}-M_{0}\right\|$. By construction, $D_{1}$ and $V_{0}$ are chosen independently at waypoints. Thus they are independent under the Palm probability.

The intensity is finite if and only if $\mathbb{E}^{0}\left(\frac{1}{V_{0}}\right)$ is finite, which, for the uniform speed case, means $v_{\min }>0$. If $v_{\min }=0$, the formula would give $\lambda=0$, which means that there is a problem. We discuss this in detail in Section 5 where we show that $v_{\text {min }}>0$ is a necessary and sufficient condition for the random waypoint model to have a stationary regime, when $f_{V}^{0}$ is uniform.

There exists a closed form for $\bar{\Delta}$ when $A$ is a rectangle or a disk [4, 5]. For a square a size $a$, $\bar{\Delta} \approx 0.5214 a$; for a disk of radius $a, \bar{\Delta} \approx 0.9054 a$. For an arbitrary area $A$, it is generally not possible to obtain $\bar{\Delta}(A)$ in closed form, but it can be obtained directly by Monte Carlo simulation. This means drawing a large number of points at random in a rectangle that contains $A$; an estimate of $\bar{\Delta}$ is the average distance between point couples that both fall in $A$. However, as we show later, we do not need to know $\bar{\Delta}$ in practice.

\subsection{Different Speed Distributions}

The Palm distribution of the speed is given by the density $f_{V}^{0}$. The time stationary distribution can be obtained from the inversion formula Equation (7).

Theorem 4.2 [14] The time stationary distribution of the numerical speed has a density proportional to $\frac{1}{v} f_{V}^{0}(v)$

This explains the histogram in Figure 2. 
Proof. The distribution of the instantaneous (numeric) speed $V(t)$ is obtained if we know $\mathbb{E}(\phi(V(t))$ for any bounded function $\phi$. We have

$$
\begin{aligned}
\mathbb{E}(\phi(V(t)) & =\lambda \mathbb{E}^{0}\left(\int_{0}^{T_{1}} \phi(V(t)) d t\right) \\
& =\lambda \mathbb{E}^{0}\left(T_{1} \phi\left(V_{0}\right)\right)=\lambda \mathbb{E}^{0}\left(\frac{D_{1}}{V_{0}} \phi\left(V_{0}\right)\right)=\lambda \mathbb{E}^{0}\left(D_{1}\right) \mathbb{E}^{0}\left(\frac{1}{V_{0}} \phi\left(V_{0}\right)\right) \\
& =K_{1} \int \frac{1}{v} \phi(v) f_{V}^{0}(v) d v
\end{aligned}
$$

where $K_{1}$ is some constant.

Obtaining a Uniform Time Stationary Distribution of Speeds This can easily be obtained from Equation (10). The distribution of the speed selected at a waypoint should be

$$
f_{V}^{0}(v)=C v 1_{\left\{v_{\min }<v<v_{\max }\right\}}
$$

i.e. with a density proportional to $v$. Such a speed is easy to simulate, by the classical method of inversion. Figure 4.2 shows simulation results with such a speed.

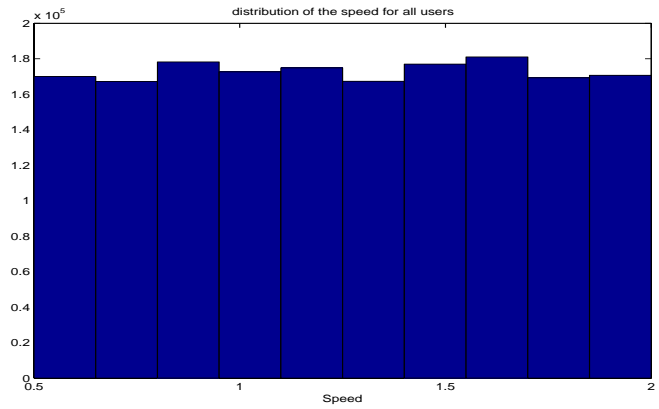

(a) Arbitrary Time Instant

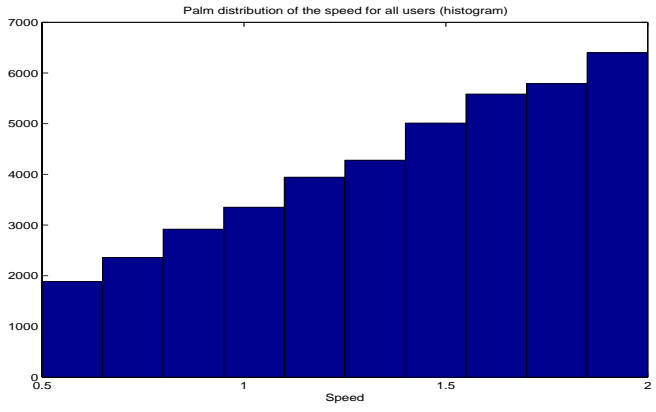

(b) Arbitrary Waypoint

Figure 5: Histogram of speeds sampled uniformly over one day (a) or at waypoints (b). Same parameters as Figure 2 except for distribution of speed at waypoints, which has a density proportional to $v$.

\subsection{Different Mobile Positions}

The Palm distribution of the mobile position $M(t)$ is uniform in the area $A$, but the time stationary distribution is not (Figure 3). So far, there did not appear to be any closed form the density $f_{M}$ of the time stationary mobile position, (except in the case where $A$ is the line segment $[0, a][4]$ in which case it has density $\left.f_{M}(x)=-\frac{6}{a^{3}} x^{2}+\frac{6}{a^{2}} x\right)$. An approximation is given in [4] for the case of a square or a disk. However, as we show now, the time stationary distribution of mobile position can still be obtained and simulated easily in closed form. Call $\operatorname{Prev}(t)$ the previous waypoint before or at time $t$ and $\operatorname{Next}(t)$ the next waypoint after time $t$ (Figure 6). By the same token as Feller's 


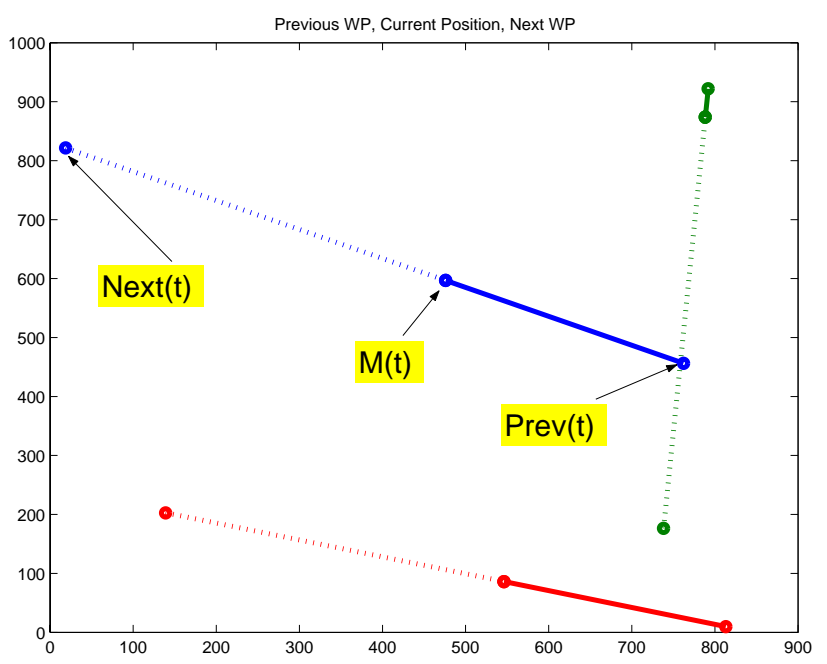

Figure 6: Definition of previous, next waypoints and current position.

paradox, the time stationary distribution of $\operatorname{Prev}(t)$ and $\operatorname{Next}(t)$ is not uniform in $A$, even though the Palm distribution is. Indeed, at an arbitrary point in time, it is more likely to find $M(t)$ in a large segment than a small one, and it is more likely that the previous and next waypoints are close to the edge than the center. See Figure 7. Again, the inversion formula gives the distribution of the

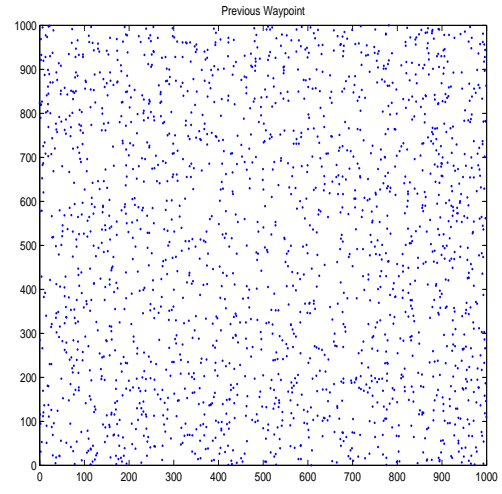

(a) $\operatorname{Prev}(t)$

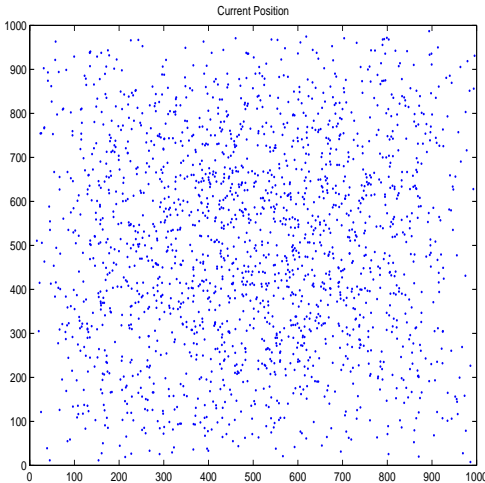

(b) $M(t)$

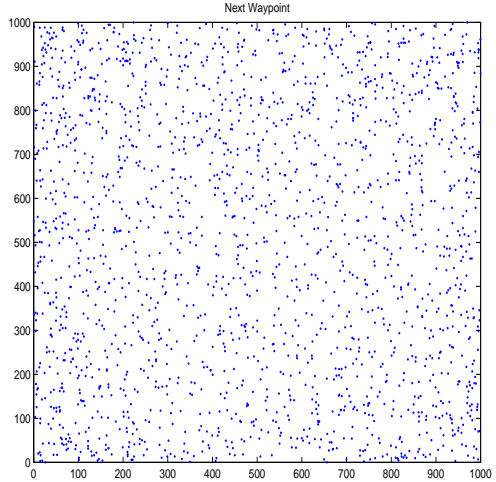

(c) $\operatorname{Prev}(t)$

Figure 7: Simulation of the time stationary distribution of previous, next waypoints and current position.

triple $(\operatorname{Prev}(t), M(t), \operatorname{Next}(t))[12]$ : 
Theorem 4.3 [12] The time stationary distribution of $((\operatorname{Prev}(t), M(t), \operatorname{Next}(t))$ has the following closed form

1. $((\operatorname{Prev}(t), \operatorname{Next}(t))$ has a joint density over $A \times A$ given by

$$
f_{\operatorname{Prev}(t), \operatorname{Next}(t)}(p, n)=K_{2}\|p-n\|
$$

where $K_{2}$ is some constant.

2. The distribution of $M(t)$ given $\operatorname{Prev}(t)=p, \operatorname{Next}(t)=n$ is uniform on the segment $[p, n]$

Proof. For any bounded, non negative function $\phi$ :

$$
\mathbb{E}(\phi(\operatorname{Prev}(t), M(t), N \operatorname{ext}(t)))=\lambda \mathbb{E}^{0}\left(\int_{0}^{T_{1}} \phi\left(M_{0}, M_{0}+\frac{t}{T_{1}}\left(M_{1}-M_{0}\right), M_{1}\right) d t\right)
$$

By a simple change of variable in the integral, we obtain

$$
\lambda \mathbb{E}^{0}\left(T_{1} \int_{0}^{1} \phi\left(M_{0}, M_{0}+u\left(M_{1}-M_{0}\right), M_{1}\right) d u\right)
$$

Now given that there is an arrival at time $0, T_{1}=\frac{\left\|M_{1}-M_{0}\right\|}{V_{0}}$ and the speed $V_{0}$ is independent of the waypoints $M_{0}$ and $M_{1}$ thus

$$
\begin{aligned}
& =\lambda \mathbb{E}^{0}\left(\frac{1}{V_{0}}\right) \mathbb{E}^{0}\left(\left\|M_{1}-M_{0}\right\| \int_{0}^{1} \phi\left(M_{0}, M_{0}+u\left(M_{1}-M_{0}\right), M_{1}\right) d u\right) \\
& =K_{2} \int_{A} \int_{A} \int_{0}^{1} \phi\left(M_{0},(1-u) M_{0}+u M_{1}, M_{1}\right)\left\|M_{1}-M_{0}\right\| d u d M_{0} d M_{1}
\end{aligned}
$$

which shows the statement.

Note that the result is valid for any convex and bounded area $A$. As we show later, there is no need to know the value of $K_{2}$ to use the theorem in a simulation.

However for completeness, let us mention that $K_{2}^{-1}=\operatorname{area}(A)^{2} \bar{\Delta}$ where area $(A)$ is the area of $A$ (in square meters). For a square a size $a, K_{2}^{-1} \approx 0.5214 a^{5}$; for a disk of radius $a, K_{2}^{-1} \approx 0.9054 \pi^{2} a^{5}$. For an arbitrary area $A$, it is generally not possible to obtain either area $(A)$ or $\bar{\Delta}(A)$ in closed form, but $K_{2}^{-1}$ can be obtained directly by Monte Carlo simulation. This means drawing a large number of points at random in a rectangle that contains $A$; an estimate of $K_{2}^{-1}$ is the average distance between points $\times$ the proportion of points that fall in $A \times$ the area of the surrounding rectangle.

Probability Density of Node Location We can derive from Theorem 4.3 some expression for the density of the time-stationary distribution of node location $M(t)$, as well as for the previous or next waypoints $\operatorname{Prev}(t)$ and $N \operatorname{ext}(t)$. 


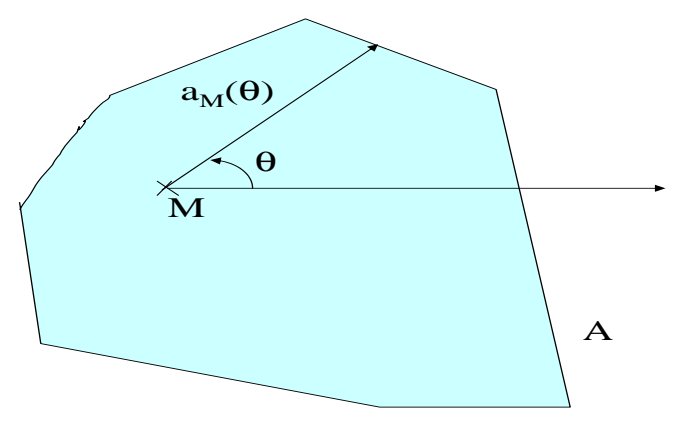

Figure 8: Definition of $a_{M}(\theta)$ in Theorem 4.4

Theorem 4.4 The probability density of the time-stationary distribution of node location $M(t)$ is

$$
f_{M(t)}(M)=K_{2} \int_{0}^{\pi} a_{M}(\theta) a_{M}(\theta+\pi)\left(a_{M}(\theta)+a_{M}(\theta+\pi)\right) d \theta
$$

where $a_{M}(\theta)$ is equal to the distance from the point $M \in A$ to the boundary of $A$, in the direction that makes an angle $\theta$ with respect to some arbitrary fixed direction (Figure 8) and $K_{2}$ is defined in Theorem 4.3.

Proof. To compute the density, we compute $\mathbb{E}(f(M(t)))$ for some arbitrary, bounded function $f$ defined on $A$. By Theorem 4.3

$$
\mathbb{E}(f(M(t)))=K_{2} \int_{A \times A \times[0,1]} f(u p+(1-u) n)\|p-n\| d p d n d u
$$

We transform the (5-dimensional) integration variable $(p, n, u)$ to $(x, y, \alpha, \beta, \theta)$ defined by

$$
\left\{\begin{array}{l}
p=(x+\alpha \cos \theta, y+\alpha \sin \theta) \\
n=(x-\beta \cos \theta, y-\beta \sin \theta) \\
u=\frac{\beta}{\alpha+\beta}
\end{array}\right.
$$

It can easily be seen that this transformation is invertible and $(x, y)=u p+(1-u) p$, so that $(x, y)$ represents the coordinates of the point $M(t)$. Further, $\|p-n\|=\alpha+\beta$. The Jacobian of this transformation can be shown to be equal to 1 . Thus:

$$
\mathbb{E}(f(M(t)))=K_{2} \int_{(x, y) \in A, \theta \in[0,2 \pi], 0 \leq \alpha \leq a_{x, y}(\theta+\pi), 0 \leq \beta \leq a_{x, y}(\theta)} f(x, y)(\alpha+\beta) d x d y d \alpha d \beta d \theta
$$

Integrate first with respect to $\alpha$ and $\beta$, then $\theta$ and obtain

$$
\mathbb{E}(f(M(t)))=K_{2} \int_{(x, y) \in A} \int_{0}^{2 \pi} f(x, y) \frac{1}{2}\left[a_{x, y}(\theta) a_{x, y}(\theta+\pi)\left(a_{x, y}(\theta)+a_{x, y}(\theta+\pi)\right)\right] d \theta d x d y
$$

The integrand is periodic with period $\pi$ thus

$$
\mathbb{E}(f(M(t)))=K_{2} \int_{(x, y) \in A} f(x, y)\left(\int_{0}^{\pi} a_{x, y}(\theta) a_{x, y}(\theta+\pi)\left(a_{x, y}(\theta)+a_{x, y}(\theta+\pi)\right) d \theta\right) d x d y
$$


Corollary 4.1 (Square Area) On the area $A=[-1 ; 1] \times[-1,1]$, the probability density of the time-stationary distribution of node location $f_{M(t)}(M)=f_{M(t)}(x, y)$ is given by

$$
\left\{\begin{array}{l}
f_{M(t)}(x, y)=f_{M(t)}(|x|,|y|) \\
\text { if }|x|<|y| \text { then } f_{M(t)}(x, y)=f_{M(t)}(|y|,|x|) \\
\text { if } 0 \leq y \leq x \text { then } f_{M(t)}(x, y)=\frac{15}{32(\sqrt{2}+2+5 \ln (1+\sqrt{2}))} F(x, y)
\end{array}\right.
$$

with $F(x, y)=$

$$
\begin{array}{rrrrrr} 
& (1-x)(2+x)(1-y) & \sqrt{1+\frac{(1-y)^{2}}{(1+x)^{2}}} & + & (1-x)(1-y)(2+y) & \sqrt{1+\frac{(1-x)^{2}}{(1+y)^{2}}} \\
+ & (1-x)(2+x)(1+y) & \sqrt{1+\frac{(1+y)^{2}}{(1+x)^{2}}} & + & (1-x)(1+y)(2-y) & \sqrt{1+\frac{(1-x)^{2}}{(1-y)^{2}}} \\
- & \frac{(1-x)^{2}(1-y)^{2}}{1+x} & \sqrt{1+\frac{(1+x)^{2}}{(1-y)^{2}}} & - & \frac{(1-x)^{2}(1-y)^{2}}{1+y} & \sqrt{1+\frac{(1+y)^{2}}{(1-x)^{2}}} \\
- & \frac{(1-x)^{2}(1+y)^{2}}{1+x} & \sqrt{1+\frac{(1+x)^{2}}{(1+y)^{2}}} & - & \frac{(1-x)^{2}(1+y)^{2}}{1-y} & \sqrt{1+\frac{(1-y)^{2}}{(1-x)^{2}}} \\
+ & (1-x)\left[1+x-(1-y)^{2}\right] & \sinh ^{-1}\left(\frac{1-y}{1+x}\right) & + & (1-y)\left[1+y-(1-x)^{2}\right] & \sinh ^{-1}\left(\frac{1-x}{1+y}\right) \\
+ & (1-x)\left[1+x-(1+y)^{2}\right] & \sinh ^{-1}\left(\frac{1+y}{1+x}\right) & + & (1+y)\left[1-y-(1-x)^{2}\right] & \sinh ^{-1}\left(\frac{1-x}{1-y}\right) \\
+ & (1-x)^{2}(1-y) & \sinh ^{-1}\left(\frac{1+x}{1-y}\right) & + & (1-x)(1-y)^{2} & \sinh ^{-1}\left(\frac{1+y}{1-x}\right) \\
+ & (1-x)^{2}(1+y) & \sinh ^{-1}\left(\frac{1+x}{1+y}\right) & + & (1-x)(1+y)^{2} & \sinh ^{-1}\left(\frac{1-y}{1-x}\right)
\end{array}
$$

where $\sinh ^{-1}(t)=\ln \left(t+\sqrt{1+t^{2}}\right)$ (inverse hyperbolic sine).

Proof. It is sufficient to consider a point $M=(x, y)$ such that $0 \leq y \leq x<1$. The integral in Theorem 4.4 can be interpreted as an integral along half the boundary of the square; instead of using the parameter $\theta$, use as parameter the $y$ [resp. $x$ ] coordinate for the portion of the boundary that is parallel to the $y$-axis [resp. $x$-axis], and do the corresponding changes in the integral. The resulting integrals have a closed form that can be found with Mathematica. The constant before $F$ is $K_{2}$ of Theorem 4.3.

In [4], the following approximation is given:

$$
\begin{aligned}
f_{M(t)}(x, y) & \approx \frac{3}{8}\left(-8 x+\frac{(x-1)\left(y^{2}+1\right)\left(x^{2}+y^{2}-2\right)}{(x+1)\left(y^{2}-1\right)}\right. \\
+ & \left.(x-1)\left(\left(-y^{2}+x-2\right) \log \left(-\frac{x+1}{x-1}\right)-(x-3) y \log \left(-\frac{y+1}{y-1}\right)\right)+8\right)
\end{aligned}
$$

It differs from the true value by up to $10 \%$ (the largest inaccuracy is at points close to the boundary). 
Corollary 4.2 (Disk) On the unit disk $A=\left\{M=(x, y): x^{2}+y^{2} \leq 1\right\}$, the probability density of the time-stationary distribution of node location is

$$
f_{M(t)}(x, y)=\frac{45}{32 \pi}\left(1-r^{2}\right) E\left(r^{2}\right)
$$

where $r=\sqrt{x^{2}+y^{2}}$ and $E\left(r^{2}\right)$ is the complete elliptic integral of the second kind, defined by

$$
E\left(r^{2}\right)=\int_{0}^{\frac{\pi}{2}} \sqrt{1-r^{2} \sin ^{2} \theta} d \theta
$$

Proof. By symmetry, the density depends only on $r$. The integral in Theorem 4.4 can be reduced to an elliptic integral after a few manipulations. The constant at the beginning of the formula derives from $\bar{\Delta}=\frac{128}{45 \pi}[4]$.

The elliptic integral of the second kind is a special function that is well tabulated and easy to compute numerically. In [4], the approximation $f_{M(t)}(x, y) \approx \frac{2}{\pi}\left(1-r^{2}\right)$ is given. It also differs from the true value by up to $10 \%$.

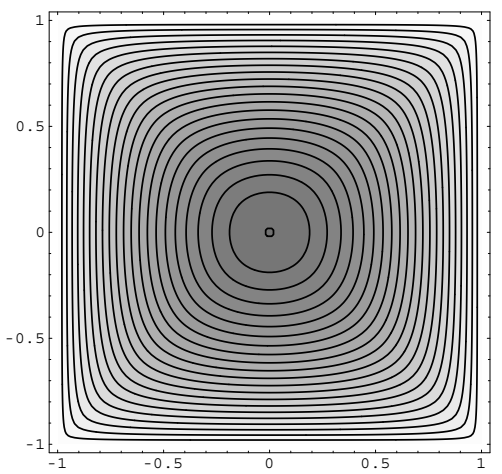

(a) Node Location

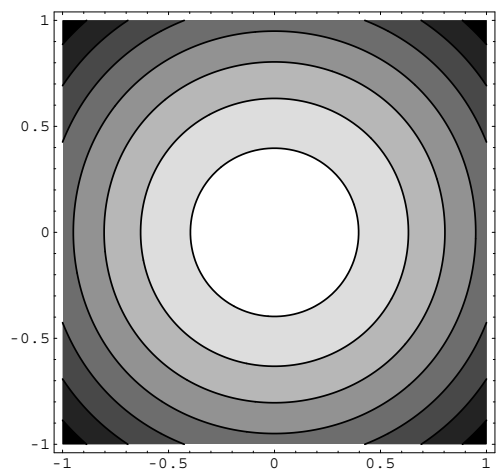

(b) Next Waypoint

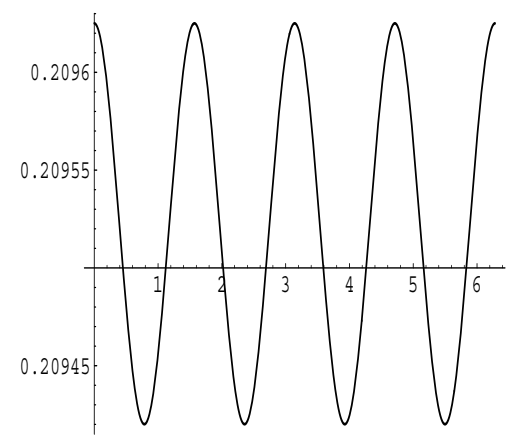

(c) Next Waypoint

Figure 9: Density of the time-stationary distribution of (a) node location $M(t)$ and (b) the previous (or next) waypoint. The contour plots (a) and (b) show lines of equal density. The difference in density between two adjacent lines is 0.025 . In (a) the density decreases from 0.5503 (center) to 0 (edge); in (b) it increases from 0.1834 to 0.3669 . The density in (b) appears to have circular symmetry, but this is not exactly true: (c) plots, for the same case as (b), the density as a function of $\theta$ for points of the form $x=0.5 \cos (\theta), y=0.5 \sin (\theta)$ (i.e. points that are at a fixed distance $r=0.5$ from the center).

Similarly, one can find that the pdf of the next waypoint $N \operatorname{ext}(t)$ (which is the same as that of Prev $(t))$ is

$$
f_{N e x t(t)}(M)=\frac{K_{2}}{3} \int_{0}^{2 \pi} a_{M}(\theta)^{3} d \theta
$$


For $(x, y)$ in the square $A=[-1 ; 1] \times[-1,1]$ we find

$$
f_{N e x t(t)}(x, y)=\frac{5}{32(\sqrt{2}+2+5 \ln (1+\sqrt{2}))}(I(x, y)+I(y, x)+I(-x,-y)+I(-y, x))
$$

with $I(x, y)=$

$$
\begin{aligned}
& \frac{1}{2}(1-x)\left[-2 \log (1-x)(x-1)^{2}+\log (-y+\sqrt{(x-2) x+(y-2) y+2}+1)\right. \\
+ & \log (y+\sqrt{(x-2) x+y(y+2)+2}+1) \\
+ & (x-2) x \log ((-y+\sqrt{(x-2) x+(y-2) y+2}+1)(y+\sqrt{(x-2) x+y(y+2)+2}+1)) \\
- & y \sqrt{(x-2) x+(y-2) y+2}+\sqrt{(x-2) x+(y-2) y+2} \\
+ & y \sqrt{(x-2) x+y(y+2)+2}+\sqrt{(x-2) x+y(y+2)+2}]
\end{aligned}
$$

For the unit disk we find

$$
f_{N e x t(t)}(x, y)=\frac{45}{32 \pi}\left(2 r^{2} \sqrt{1-r^{2}}+2 r \arcsin (r)-\left(1-r^{2}\right) E\left(r^{2}\right)\right)
$$

with $r$ and $E$ as defined in Corollary 4.2.

Note that the closed forms in Equations (14) to (18) are not helpful for simulation. As we show next, it is better to use the form in Theorem 4.3, as we need the joint distribution - and as it is much simpler.

\subsection{Perfect Simulation}

"Perfect simulation" means a simulation without transients. It is possible if we know the time stationary distribution of the simulation state. For the random waypoint, the state of the simulation can be taken as $S_{t}=(M(t), V(t), \operatorname{Next}(t))$. Note that knowing the time stationary distribution of the mobile position is not sufficient for perfect simulation, contrary to what is used in the literature. We already know the distribution of $(M(t), \operatorname{Next}(t))$, it remains to see how to use Theorem 4.3 in order to simulate the time stationary position of mobiles. The only technical issue is to draw a couple of points $\left(M_{0}, M_{1}\right)$ in $A \times A$ with density $\left\|M_{1}-M_{0}\right\|$.

Theorem 4.5 The time stationary distribution of the previous and next waypoint and the current mobile position can be obtained as follows. Let $\Delta$ be an upper bound on the diameter of area $A$.

1. do

draw $M_{0}, M_{1}$ iid $\sim \operatorname{Unif}(A)$

draw $V \sim \operatorname{Unif}[0, \Delta]$

until $V<\left\|M_{1}-M_{0}\right\|$

$\operatorname{Prev}(t)=M_{0}$ and $\operatorname{Next}(t)=M_{1}$

2. Draw $U \sim$ Unif $[0,1]$

3. $M(t)=(1-U) M_{0}+U M_{1}$ 
Proof. A generic method to simulate a random vector $X$ with bounded density $f_{X}$ over a bounded area $A$ (not necessarily convex) is as follows [13]. Find an upper bound $H$ on the density $f_{X}$. Generate $x$ uniform in the area $A$ and $v$ uniform in the set of real numbers $[0, H]$. Accept $x$ if $v \leq f_{X}(x)$ else start again. The rest is a straightforward application of this method to Theorem 4.3.

The idea of the theorem is simple: draw $M_{0}, M_{1}$ random in $A$ and measure their distance $\left\|M_{1}-M_{0}\right\|$. Keep them with probability $\left\|M_{1}-M_{0}\right\| / \Delta$. This requires a variable number of iterations, but in average, this number is equal to $\Delta / \bar{\Delta}(A)$, and thus is very small $(=2.71$ for a square area, $=2.21$ for a disk). There is no need to know the constant $K_{2}$ of the exact distribution of the node position, nor is there a need to have an exact value for the diameter of $A$ (an upper bound is enough).

The remaining part is to know how to sample jointly speed and locations; this is given by the following theorem. Again, it is a direct consequence of the inversion formula.

Theorem 4.6 The numerical speed $V(t)$ and $(M(t), N e x t(t))$ are independent under the time stationary distribution.

Proof. Consider two bounded, arbitrary functions $\phi$ and $\psi$. By the inversion formula:

$$
\begin{aligned}
\mathbb{E} & (\phi(V(t)) \psi(M(t), \operatorname{Next}(t))) \\
& \left.=\lambda \mathbb{E}^{0}\left(\int_{0}^{T_{1}} \phi\left(V_{0}\right)\right) \psi\left(M_{0}+\frac{t}{T_{1}}\left(M_{1}-M_{0}\right), M_{1}\right) d t\right) \\
& =\lambda \mathbb{E}^{0}\left(\frac{\phi\left(V_{0}\right)}{V_{0}}\left\|M_{1}-M_{0}\right\| \int_{0}^{1} \psi\left(M_{0}+t\left(M_{1}-M_{0}\right), M_{1}\right) d t\right) \\
& =\lambda \mathbb{E}^{0}\left(\frac{\phi\left(V_{0}\right)}{V_{0}}\right) \mathbb{E}^{0}\left(\left\|M_{1}-M_{0}\right\| \int_{0}^{1} \psi\left(M_{0}+t\left(M_{1}-M_{0}\right), M_{1}\right) d t\right)
\end{aligned}
$$

Thus we have factored the joint expectation in two terms, which proves independence (see Lemma in the appendix of [6]).

We are now able to formulate a perfect simulation method. The following theorem gathers the results in this section.

Theorem 4.7 (Perfect Simulation) Consider a random waypoint simulation, initialized at time 0 as follows.

1. Sample $(p, m, n)$ from time stationary distribution of $(\operatorname{Prev}(t), M(t), \operatorname{Next}(t))$ (using Theorem 4.5)

2. Sample $v$ from the time stationary distribution of $V(t)$ (using Theorem 4.2)

3. Start the simulation with initial position $M(0)=m$, speed $V(0)=v$ and next waypoint $=n$.

The simulation is in stationary regime at any time $t \geq 0$.

Note that the first waypoint of the simulation is obtained by the initialization procedure, not by 
drawing a point uniformly in $A$. In contrast, after the first waypoint is reached, the next waypoint is chosen uniformly in $A$, as in Section 2.1. Figure 10 illustrates that there is no speed decay with a perfect simulation.

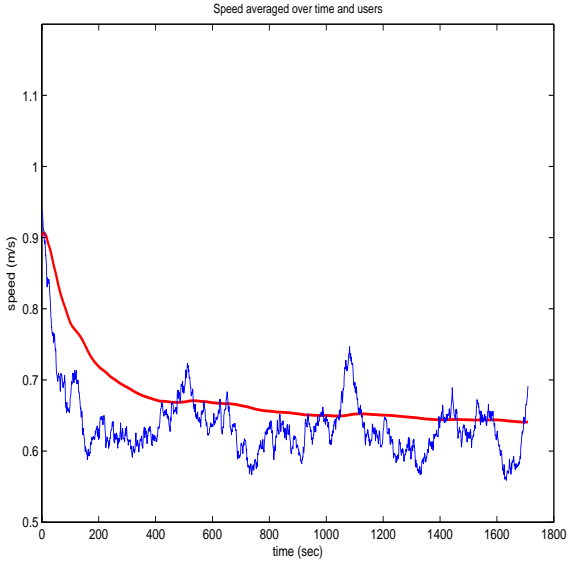

(a) Standard Initialization

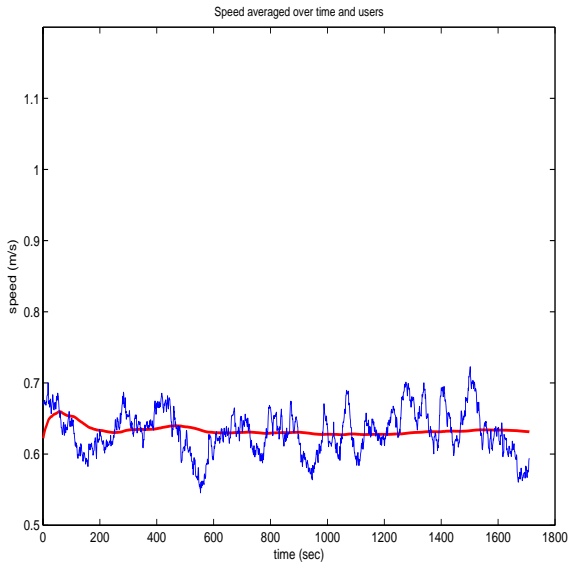

(b) Initialization as in Theorem 4.7 (perfect simulation

Figure 10: Standard versus perfect simulation of the random waypoint. Same parameters as Figure 2 except $v_{\min }=0.1 \mathrm{~m} / \mathrm{s}$. The irregular line is the instant speed averaged over 200 mobiles; it has no decay with perfect simulation. The smooth line is the running average of the speed (distance divided by time) and converges in both cases to the time stationary expectation of the speed.

\section{Stability of Random Waypoint}

In Equation (9), we found that we need that $\mathbb{E}^{0}\left(\frac{1}{V_{0}}\right)$ be finite for a stationary regime to exist. We now discuss when the random waypoint model indeed has a stationary regime. We first consider both the theoretical model and what really happens in a simulator.

\subsection{Theoretical Model}

The theoretical model has a continuous set of values for speed and positions. As a result, is is well possible that $\mathbb{E}^{0}\left(\frac{1}{V_{0}}\right)=\infty$. This occurs for example with the uniform speed model when $v_{\min }=0$. We have the following result. Although very intuitive, its proof is complex and is not given here (see [2]; it relies on the so-called "inverse construction" in [1]).

Theorem 5.1 The random waypoint has a stationary regime if and only if $\mathbb{E}^{0}\left(\frac{1}{V_{0}}\right)<\infty$

It follows immediately that, with uniform speed, the model has a stationary regime if and only if $v_{\min }>0$.

Note that if we choose the Palm distribution of speed $f_{V}^{0}$ such that the stationary distribution of speed is uniform (Equation (11)), then the model always has a stationary regime, even for $v_{\min }=0$. 
When the model does not have a stationary regime, it can be shown that the empirical time speed average (distance run divided by time) converges to 0 . This is the behaviour of Figure 4 (b). Thus the decay in this case can be interpreted as lack of convergence to a stationary regime.

\subsection{Practical Model}

If we want to understand what really happens in a computer simulation of the random waypoint, we nedd to consider that the state space of the simulation is finite (because there are only a finite number or memory states). In such a case, instability of the simulation cannot occur. The simulator always converges to some stationary regime.

To understand more in detail what this stationary regime is, we focus on the uniform speed model with $v_{\min }=0$ (i.e. the case where the theoretical model is unstable but the practical one is). At every waypoint, the simulator picks a random speed uniform in $\left[0, v_{\max }\right]$. In reality, this means that a number is picked uniformly in the finite set

$$
\left\{v_{\min }+\epsilon, v_{\min }+2 \epsilon, \ldots, v_{\max }-2 \epsilon, v_{\max }-\epsilon\right\}
$$

where $\epsilon$ is the granularity of the random number generator [11]. For this model, we find

$$
\mathbb{E}^{0}\left(\frac{1}{V_{0}}\right) \approx \frac{1}{v_{\max }-v_{\min }} \int_{v_{\min }+\epsilon}^{v_{\max }-\epsilon} \frac{d v}{v}=\frac{\ln \left(v_{\max }-\epsilon\right)-\ln \left(v_{\min }+\epsilon\right)}{v_{\max }-v_{\min }}
$$

and thus for

$$
\lambda=O\left(\frac{1}{-\ln \epsilon}\right) \text { for } \epsilon \rightarrow 0
$$

Thus, in reality, the practical model has a stationary regime, but the intensity that decays very slowly with the accuracy of the simulator.

\section{More General Random Waypoint Models}

In this section we describe variants of the random waypoint model in Section 2.1. The results and methods in the previous sections extend directly to these variants, therefore we mention most results without the details of the proofs.

\subsection{Random Waypoint With Pauses}

Consider the same model as in Section 2.1, with the following modification.

- When a mobile reaches a waypoint, it draws a random duration from the density $f_{\text {pa }}^{0}$, stays immobile for this duration, and then continues as before.

To analyze this model, we consider as selected transition times the times at which either a waypoint is reached or a pause time is finished. We call $\Phi(t)$ the phase of the mobile at time $t$, with $\Phi(t)=p a$ 
or $\Phi(t)=m o$. Also define $\Phi_{n}$ as the phase chosen at the $n$th transition, i.e. $\Phi(t)=\Phi_{n}$ for $T_{n} \leq t<T_{n+1}$. $\Phi_{n}$ is a Markov chain with two states and in steady state has equal probability of being in either state:

$$
\mathbb{P}^{0}\left(\Phi_{0}=p a\right)=\mathbb{P}^{0}\left(\Phi_{0}=m o\right)=0.5
$$

The notation is otherwise the same as for the random waypoint without pause. In particular, $M_{0}$ is the position at time $T_{0}, M_{1}$ at time $T_{1}$, and $M_{1}=M_{0}$ if $\Phi_{0}=p a$.

$\mathbb{P}^{0}$ is now the event stationary probability at an arbitrary transition. We also introduce the event stationary probability at the beginning of a pause [resp. move] interval. These are simple conditional expectations: for any observable process $X_{t}$ that is jointly stationary with the simulation:

$$
\mathbb{E}_{p a}^{0}\left(X_{0}\right)=\frac{\mathbb{E}_{p a}^{0}\left(X_{0} 1_{\left\{\Phi_{0}=p a\right\}}\right)}{\mathbb{P}^{0}\left(\Phi_{0}=p a\right)}
$$

and similarly for $\mathbb{E}_{m o}^{0}$. Thus for example the mean duration between transitions, observed in a pause interval is $\mathbb{E}_{p a}^{0}\left(T_{1}\right)$.

Intensities The intensity formula gives

\section{Theorem 6.1}

$$
\frac{1}{\lambda}=0.5 \frac{1}{\lambda_{p a}}+0.5 \frac{1}{\lambda_{m o}}
$$

with

$$
\begin{aligned}
\frac{1}{\lambda_{p a}} & =\mathbb{E}_{p a}^{0}\left(T_{1}\right)=\int_{0}^{\infty} f_{p a}^{0}(t) d t \\
\frac{1}{\lambda_{m o}}=\mathbb{E}_{m o}^{0}\left(T_{1}\right) & =E_{m o}^{0}\left(D_{1}\right) \mathbb{E}_{m o}^{0}\left(\frac{1}{V_{0}}\right)=\int_{0}^{\infty} \frac{f_{V}^{0}(v)}{v} d v \bar{\Delta}
\end{aligned}
$$

Thus $\lambda_{m o}$ is equal to the intensity of waypoints in the model without pause and $\lambda_{p a}$ is the average number of pauses per time unit in a hypothetical model that would not move but would pause.

In contrast, the average number of pauses per time unit in this model is $0.5 \lambda$ and the average number of moves per time unit is also $0.5 \lambda$.

\section{Time Stationary Distributions}




$$
\left\{\begin{array}{l}
\mathbb{P}(I(t)=p a))=0.5 \frac{\lambda}{\lambda_{p a}}=\frac{1 / \lambda_{p a}}{1 / \lambda_{p a}+1 / \lambda_{m o}} \\
\mathbb{P}(I(t)=m o))=0.5 \frac{\lambda}{\lambda_{m o}}=\frac{1 / \lambda_{m o}}{1 / \lambda_{p a}+1 / \lambda_{m o}}
\end{array}\right.
$$

- Under the time stationary distribution conditional to being in a pause phase, the position and the time until the end of pause are independent. The former is uniform in A; the latter has density $\lambda_{p a} \int_{t}^{\infty} f_{p a}^{0}(s) d s$.

- The time stationary distribution of speed and position, conditional to being in a move phase, are given by Theorem 4.2 and Theorem 4.3, where we replace "time stationary distribution" by "time stationary distribution conditional to being in a mo phase".

Proof. Apply the inversion formula to the indicator function $1_{\{I(t)=p a\}}$ (first item) or, for example, $V(t) 1_{\{I(t)=m o\}}$ (second item).

The theorem completely describes the time stationary behaviour of the random waypoint with pauses. The phase of the model is distributed proportional to the average duration spent in a phase. The theorem involves the average distance $\bar{\Delta}$, which, as mentioned earlier, is known exactly only for simple areas $A$. However, as we show next, it is possible to avoid knowing $\bar{\Delta}$ to simulate the time stationary distribution.

Perfect Simulation Define $\alpha$ by

$$
\frac{1}{\alpha}=\lambda_{p a} \int_{0}^{\infty} \frac{1}{v} f_{V}^{0}(v) d v
$$

Thus $\alpha$ is a distance proportional to the time stationary speed and the average pause time. The time stationary probability $q$ to be in a move phase is equal to $\frac{1 / \lambda_{p a}}{1 / \lambda_{p a}+1 / \lambda_{m o}}$. After some algebra, we can write also:

$$
q=\frac{\alpha}{\alpha+\bar{\Delta}}
$$

A straightforward method for perfect simulation follows from Theorem 6.2, assuming we know $\bar{\Delta}$. This occurs on simple areas for which there is a closed form, or on arbitrary area, if a preliminary Monte Carlo simulation was run to compute $\Delta$ (Section 4).

Theorem 6.3 (Perfect Simulation of Random Waypoint with Pauses, $\bar{\Delta}$ assumed known [12]) Draw $\phi=$ pa with probability $q$ else let $\phi=m o$.

2. If $\phi=p a$ sample a time $t$ from the distribution with density $\lambda_{p a} \int_{t}^{\infty} f_{p a}^{0}(s) d s$. Sample a point $m$ uniformly in $A$. Start the simulation in pause phase with initial position $M(0)=m$ and schedule the end of the pause at time $t$.

3. If $\phi=m o$ sample $(p, m, n)$ from the time stationary distribution of $(\operatorname{Prev}(t), M(t), \operatorname{Next}(t))$ (using Theorem 4.5), sample $v$ from the time stationary distribution of $V(t)$ (using Theorem 4.2). Start the simulation in move phase with initial position $M(0)=m$, speed $V(0)=v$ and next waypoint $=n$. 
There is an alternative method, as we show now, which does not require to know $\bar{\Delta}$. Let $\Delta$ be any upper bound on the diameter of $A$. Define

$$
q_{0}=\frac{\alpha}{\alpha+\Delta}
$$

Theorem 6.4 (Perfect Simulation of Random Waypoint with Pauses, $\bar{\Delta}$ unknown.) The following method provides a perfect simulation of the random waypoint with pauses.

\section{1. do forever}

$$
\begin{aligned}
& \text { Draw } \phi=\text { pa with probability } q_{0} \\
& \text { if success, decide that } \Phi=\text { a; leave } \\
& \text { else } \\
& \quad \text { draw } M_{0}, M_{1} \text { iid } \sim \operatorname{Unif}(A) \\
& \quad \text { draw } U \sim \text { Unif }[0, \Delta] \\
& \quad \text { if } U<\left\|M_{1}-M_{0}\right\| \text { decide that } \Phi=\text { mo; leave }
\end{aligned}
$$

\section{end do}

2. If $\phi=p a$ sample a time $t$ from the distribution with density $\lambda_{p a} \int_{t}^{\infty} f_{p a}^{0}(s) d s$. Sample a point $m$ uniformly in $A$. Start the simulation in pause phase with initial position $M(0)=m$ and schedule the end of the pause at time $t$.

3. If $\phi=m o$, sample $v$ from the time stationary distribution of $V(t)$ (using Theorem 4.2), sample $s$ uniform in $[0,1]$. Start the simulation in move phase with initial position $(1-$ $s) M_{0}+s M_{1}$, next waypoint $=M_{1}$ and speed $=v$.

Proof. Let $\Phi_{k}$ be the phase drawn at the $k$ iteration of the loop and $T$ be the number of iterations when we exit the loop (if ever). We have $\mathbb{P}(T=k)=q_{1}\left(1-q_{1}\right)^{k-1}$ with

$$
q_{1}=q_{0}+\left(1-q_{0}\right) \frac{\bar{\Delta}}{\Delta}=\frac{\alpha+\bar{\Delta}}{\alpha+\Delta}
$$

Note that $0<q_{1} \leq 1$ thus the loop terminates with probability 1 . $\Phi_{T}$ is the phase when we exit the loop and

$$
\begin{aligned}
\mathbb{P}\left(\Phi_{T}=p a\right) & =\sum_{k \geq 1} \mathbb{P}\left(\Phi_{T}=p a \text { and } T=k \mid T \geq k\right)\left(1-q_{1}\right)^{k-1} \\
& =\sum_{k \geq 1} q_{0}\left(1-q_{1}\right)^{k-1}=\frac{q_{0}}{q_{1}}=\frac{\alpha}{\alpha+\bar{\Delta}}
\end{aligned}
$$

Thus the algorithm draws the phase according to the time stationary distribution. The rest follows easily.

Complexity. We count the complexity of these two simulation methods in numbers of calls to the random number generator. Let $a$ be the number of such calls required to simulate one sample $\left(M_{0}, M_{1}\right)$ uniformly uniformly in the area $A$ plus one ( $a=5$ for a rectangle or a disk, more for arbitrary areas). We have

$$
C_{1}=\frac{\alpha+\Delta a}{\alpha+\bar{\Delta}}
$$


and

$$
C_{2}=\frac{\Delta-\bar{\Delta}}{\alpha+\bar{\Delta}}(1+a)+\frac{\alpha+(1+a) \Delta}{\alpha+\Delta}
$$

We always have $C_{2}>C_{1}$; the difference is negligible when $\alpha$ is small or when $a$ or $\Delta$ is large. Thus Theorem 6.4 is preferable when the domain $A$ is difficult to simulate exactly.

Conditions for Stationarity The condition is $\mathbb{E}_{m o}^{0}\left(1 / V_{0}\right)<\infty$ and $\mathbb{E}_{p a}^{0}\left(T_{1}\right)<\infty$, i.e

$$
\int_{0}^{\infty} \frac{1}{v} f_{V}^{0}(v) d v<\infty \text { and } \int_{0}^{\infty} f_{p a}^{0}(t) d t<\infty
$$

\subsection{Random Waypoint on General Connected Area}

The previous results can be extended without much modification to an arbitrary connected and bounded (not necessarily convex) area $A$ is an arbitrary connected and bounded area in $\mathbb{R}^{2}$ or $\mathbb{R}^{3}$. For two points $m, n$ in $A$, we call $d(m, n)$ the distance from $m$ to $n$ in $A$, i.e. the minimum length of a path entirely inside $A$ that connects $m$ and $n$. The random waypoint with pause is defined as usual: at the end of a trip, say at point $M_{n}$, a new point $M_{n+1}$ is chosen at random uniformly in $A$. The next trip is the shortest path in $A$ from $M_{n}$ to $M_{n+1}$. We assume to simplify that, expect for a set of points with zero mass, there is only one shortest path on $A$ from one point to another.

Here are some examples:

1. (Non convex planar area): Figure 11

2. (Sphere) Here the shortest path between two points is the shortest of the arcs on the great circle that contains the two points. If the two points are are on the same great circle diameter, the two arcs have same length but this occurs with probability 0.

The results in the previous sections hold with only the following adaptations.

- Replace $\|p-n\|$ by $d(p, n)$

- Theorem 4.3: replace "segment $[p, n]$ " by "shortest path from $p$ to $n$ "

- Theorem 4.5: replace " $M(t)=(1-U) M_{0}+U M_{1}$ " by " $M(t)$ is on the shortest path from $M_{0}$ to $M_{1}$ at a distance $U d\left(M_{0}, M_{1}\right)$ from $M_{0}$ ".

The final results for the two examples above are:

1. See Figure 11 for an illustration on a planar non convex area. The stationary distribution of the mobile location $M(t)$ is not uniform.

2. On the sphere example, the stationary distribution of the mobile location $M(t)$ is uniform. This follows easily from Theorem 4.5 , by noting that the distribution of $M(t)$ is invariant under any rotation of the sphere around an axis that contains the center of the sphere, and any distribution that has such an invariance property must be uniform. 


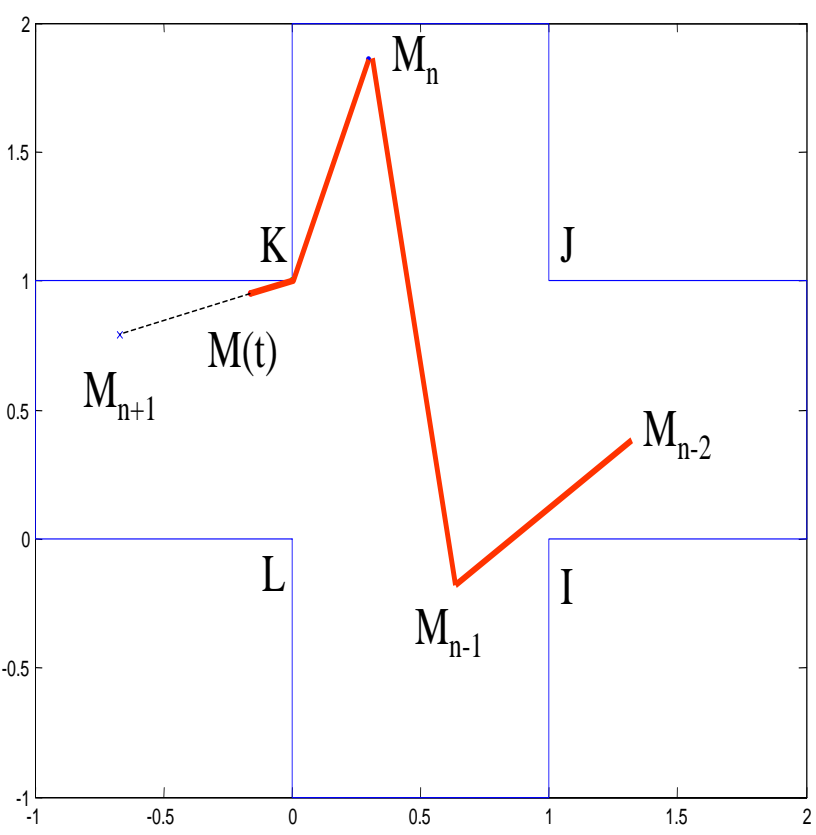

(a)

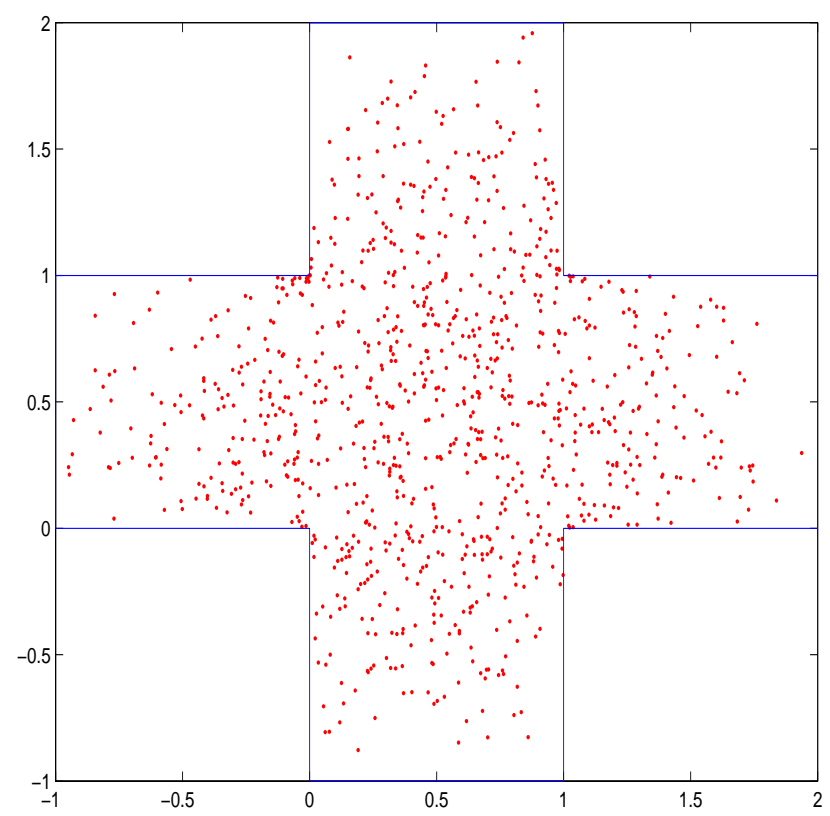

(b)

Figure 11: Random Waypoint on a non convex area. (a) A trip is the shortest path inside the area from a waypoint $M_{n}$ to the next. Waypoints $M_{n}$ are drawn uniformly in the area. On the figure, the shortest path $M_{n}, M_{n+1}$ has two segments, with a breakpoint at $K$; the shortest paths $M_{n-1}, M_{n}$ and $M_{n-2}, M_{n-1}$ have one segment each. $M(t)$ is the current position. (b) Sample of 1000 independent points from the stationary distribution of $M(t)$ : the distribution is not uniform, with a higher density towards the center and the corner points $I, J, K, L$.

\subsection{Random Walk (or Random Vector)}

This is a variant of the random waypoint defined as follows. It exists in two variants: with reflection, or with wrapping 

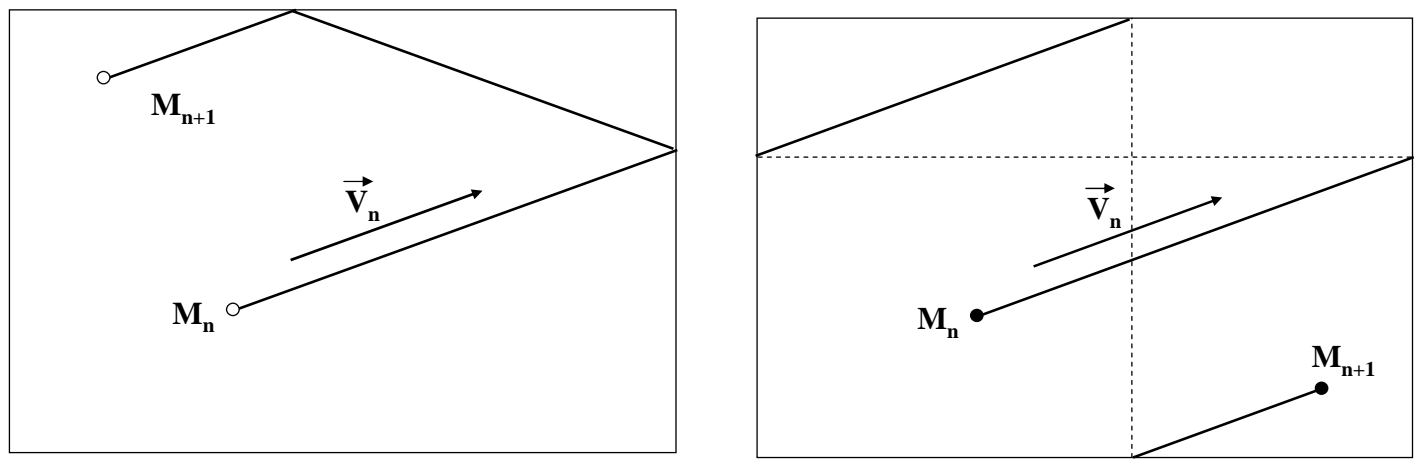

Figure 12: Definition of Random Walk with reflection and with wrapping.

- At the end of a trip, instead of choosing a next waypoint, the mobile chooses a speed vector $\vec{V}_{n}$ and a trip duration $T_{n+1}-T_{n}$ independently of the past and of each other. Choosing a speed vector $\vec{V}_{n}$ is the same as choosing a direction of movement and a numerical speed. Initially, the mobile location is chosen uniformly in $A$.

- The area $A$ is the rectangle $[0, a] \times[0, b]$. The mobile moves from the current position $M_{n}$ in the direction of the speed vector. When it hits the boundary of $A$, say for example at a location $\left(x_{0}, b\right)$, it is reflected and continues from there [resp. it is wrapped to the other side, to location $\left(x_{0}, 0\right)$ ] (Figure 12).

- The speed vector is chosen according to a density $f_{\vec{V}}^{0}(\vec{v})$ in the plane; the trip duration with density $f_{T}^{0}(t)$ over the set of positive numbers.

This model is called "Random Walk" in [3]. With wrapping, it is sometimes viewed as a random waypoint on a torus [8]. It is used primarily because of its simplicity: unlike for the random waypoint, the distribution of location and speed at a random instant are the same as at a transition instant, as we explain next. This comes from the fact that, if the location $M_{n}$ is uniform in $A$, then so is $M_{n+1}$ (as shown in the proof of the following theorem, to be found in [2]).

Theorem 6.5 [2] The random walk has a stationary regime if and only if $\int_{0}^{\infty} t f_{T}^{0}(t) d t$ is finite. If this condition is met:

- The intensity of transitions is given by $\lambda^{-1}=\int_{0}^{\infty} t f_{T}^{0}(t) d t$.

- The stationary distribution of $(M(t), \vec{V}(t), R(t))$ is given by

- $M(t)$ is uniform in $A=[0, a] \times[0, b]$

- $\vec{V}(t)$ has the density $f_{\vec{V}}^{0}(\vec{v})$

- $R(t)$ has the density $f_{R}(r)=\lambda \int_{r}^{\infty} f_{T}^{0}(s) d s=\lambda \mathbb{P}^{0}\left(T_{1}>r\right)$

- $M(t), \vec{V}(t)$ and $R(t)$ are independent.

Perfect simulation of the random walk is thus simple. Pick a point and speed vector as if at a transition point, and pick a remaining trip duration according to the density $f_{R}(r)$. Note that the formula for $f_{R}(r)$ is the general formula for the density of the time until next transition, in any stationary system. 


\section{Conclusion}

We have shown that the random waypoint model can easily be understood with a little of Palm calculus, the essentials of which we explained very briefly. Palm calculus is intuitive and easy to manipulate if we reason in discrete time. The proof of the intensity and inversion formulas, heavily used with the random waypoint, are simple and can be taught in a first course on probability [7].

The decay of the random waypoint model is, in all practical cases, simply the convergence to stationary regime, a common feature of any non terminating computer simulation. In some exceptional case (uniform speed model with $v_{\min }=0$ ) the random waypoint is theoretically unstable (has no stationary regime), but this cannot be observed in practice.

The stationary distribution of all aspects of the random waypoint model is easily obtained, thanks to the Palm inversion formula, even if no closed form exists for the density of the stationary position of a mobile. We have also shown that it is very simple to implement a perfect simulation, i.e., a simulation that has no transient, and thus no decay.

\section{References}

[1] François Baccelli and Pierre Brémaud. Palm Probabilities and Stationary Queues. Springer LNS, 1987.

[2] Jean-Yves Le Boudec and Milan Vojnović. Perfect simulation and stationarity of a class of mobility models. In Proc. of IEEE Infocom '05, March 2005.

[3] T Camp, J. Boleng, and V. Davies. A survey of mobility models for ad hoc network research. WCMC: Special issue on Mobile Ad Hoc Networking: Research, Trends and Applications, 2(5):483-502, 2002.

[4] Hannes Hartenstein Christian Bettstetter and Xavier Prez-Costa. Stochastic properties of the random waypoint mobility model. ACM/Kluwer Wireless Networks, Special Issue on Modeling and Analysis of Mobile Networks 2003.

[5] B. Ghosh. Random distances within a rectangle and between two rectangles. Bulletin Calcutta Mathematical Society, 43:17-24, 1951.

[6] J. Y. Le Boudec. On the stationary distribution of speed and location of random waypoint. IEEE transactions on mobile computing, to appear.

[7] Jean-Yves Le Boudec. Performance evaluation lecture notes. http://ica1www.epfl.ch/perfeval/perfeval.htm, March 2004.

[8] Guolong Lin, Guevara Noubir, and Rajmohan Rajamaran. Mobility models for ad-hoc network simulation. In Proceedings of Infocom 2004, April 2004.

[9] James G. Propp and David B. Wilson. Exact sampling with coupled markov chains and applications to statistical mechanics. Random Structures and Algorithms, 9(1 \& 2):223-252, 1996. 
[10] Walter Rudin. Real and Complex Analysis. McGraw-Hill Series in Mathematics, 1987.

[11] Matlab technical solutions. Solution number 8542, 2003-02-11.

[12] T. Camp W. Navidi and N. Bauer. Improving the accuracy of random waypoint simulations through steady-state initialization. In Proceedings of the 15th International Conference on Modeling and Simulation (MS '04), pages 319-326, March 2004.

[13] Bernard Ycart. Modèles et algorithmes markoviens, volume 39. Springer Verlag, 2002.

[14] Jungkeun Yoon, Mingyan Liu, and Brian Noble. Random waypoint considered harmful. In Proceedings of Infocom, 2003.

[15] Jungkeun Yoon, Mingyan Liu, and Brian Noble. Sound mobility models. In Proceedings of Mobicom, 2003. 